\title{
APLICAÇÕES TECNOLÓGICAS E ALTERNATIVAS PARA REDUÇÃO DO CLORETO DE SÓDIO EM PRODUTOS CÁRNEOS
}

\author{
CARLOS EDUARDO ROCHA GARCIA* \\ VINÍCIUS JOSÉ BOLOGNESI** \\ MASSAMI SHIMOKOMAKI ${ }^{\star \star *}$
}

\begin{abstract}
O objetivo deste trabalho foi revisar as aplicações tecnológicas e alternativas para redução do uso de cloreto de sódio em produtos cárneos. Foram abordadas as características do consumo de cloreto de sódio, destacando sua ação hipertensiva, as aplicações tecnológicas do cloreto de sódio em produtos cárneos, incluindo a promoção do gosto salgado, seu efeito sobre a atividade de água e a estabilidade microbiológica, além da sua influência nas propriedades funcionais das proteínas cárneas. Também foram apontados os métodos disponíveis para redução dos níveis de $\mathrm{NaCl}$ nos derivados cárneos, como o uso de sais não sódicos, novos ingredientes e métodos de conservação alternativos. A preocupação dos consumidores relacionada aos efeitos prejudiciais à saúde, associada ao consumo excessivo de sódio, exigirá das indústrias alimentícias continua redução do uso de sal nos alimentos. Essa limitação enfrenta maior complexidade no segmento cárneo, pois além de prover palatabilidade, o $\mathrm{NaCl}$ é responsável por diversas propriedades funcionais. A busca de alternativas para redução do uso de $\mathrm{NaCl}$, sem prejuízo para a qualidade sensorial dos produtos, constitui desafio a ser enfrentado pela indústria, visando manter a aceitação e consumo dos derivados cárneos.
\end{abstract}

PALAVRAS-CHAVE: CARNE; HIPERTENSÃO; NaCI; PROPRIEDADES FUNCIONAIS; SUBSTITUTO DO SAL.

* Doutor em Ciência de Alimentos, Docente, Departamento de Farmácia, Universidade Federal do Paraná (UFPR), Curitiba, PR, Brasil (e-mail: carlos.garcia@ufpr.br).

** Mestrando em Ciências Farmacêuticas, Setor de Ciências da Saúde, UFPR, Curitiba, PR, Brasil (e-mail: josebolognesi@yahoo.com.br).

*** Doutor em Ciência de Alimentos, Docente, Departamento de Ciência e Tecnologia de Alimentos, Universidade Estadual de Londrina (UEL), Londrina, Pr, Brasil, Docente, Universidade Tecnológica Federal do Paraná (UTFPR), Londrina, Pr, Brasil (e-mail: mshimo@uel.br). 


\section{INTRODUÇÃO}

O uso do cloreto de sódio ( $\mathrm{NaCl}$ ) ocorre com tamanha amplitude no segmento alimentício, que esse composto passou a ser reconhecido simplesmente como sal de cozinha em alusão a classe química a que pertence e se destaca pelas inúmeras aplicações tecnológicas. Embora o sódio constitua a menor fração ( $23 \mathrm{~g}$ ) da massa molecular do $\mathrm{NaCl}(58,5 \mathrm{~g})$, esse íon é o principal responsável por despertar a preocupação dos consumidores em razão dos efeitos prejudicais à saúde que acompanham seu consumo excessivo (LESSA, 2010).

A elevada concentração de sódio na dieta está relacionada ao aumento da pressão arterial e representa importante fator de risco para o desenvolvimento de doenças cardiovasculares secundárias. Segundo dados compilados e divulgados nas Diretrizes Brasileiras de Hipertensão, estudos realizados nos últimos 15 anos demonstram que a hipertensão arterial acomete aproximadamente $30 \%$ dos brasileiros e que esse percentual alcança $50 \%$ em indivíduos entre 60 e 69 anos (SBC, 2010). Diante desse cenário, torna-se crescente a preocupação das agências reguladoras em minimizar as concentrações de sódio consumidas diariamente pela população (SACN, 2003; FSAI, 2005; US DEPARTAMENT OF HEALTH AND HUMAN SERVICES, 2005).

Significativa parcela do sódio presente na dieta provém de alimentos industrializados e dentre esses se destacam os derivados cárneos. Nesses produtos, além da palatabilidade emprestada pelo gosto salgado, o cloreto de sódio é responsável pelo desenvolvimento de propriedades funcionais e influencia de forma decisiva sua estabilidade e conservação (DESMOND, 2006).

Em razão da importância do tema, este trabalho teve por objetivo revisar as aplicações tecnológicas e alternativas para redução do uso de cloreto de sódio em produtos cárneos.

\section{CARACTERÍSTICAS DO CONSUMO DE CLORETO DE SÓDIO}

O consumo de sódio pelos brasileiros alcança aproximadamente 4,5 gramas diárias, quantidade duas vezes superior à recomendada pela Organização Mundial de Saúde (IBGE, 2010; WHO, 2003). Cerca de três quartos do sódio ingerido provém do sal de cozinha adicionado diretamente aos alimentos $(71,5 \%)$ ou de condimentos formulados com cloreto de sódio $(4,7 \%)$ (Tabela 1), enquanto o restante é ofertado por meio de alimentos processados (Tabela 2) contendo esse ingrediente com fins organolépticos ou tecnológicos (SARNO et al., 2009).

A redução do consumo de $\mathrm{NaCl}$ está associada, além da prática intencional de minimizar seu uso nas preparações culinárias, à oferta de produtos industrializados com menor concentração de sódio. Os produtos cárneos são significativas fontes de sódio em razão das suas aplicações tecnológicas visando a palatabilidade e o desenvolvimento de propriedades funcionais (RUUSUNEN e PUOLANNE, 2005; DESMOND, 2006). O teor de sódio nesses produtos pode superar facilmente concentrações entre $1 \%$ e $2 \%$, valores expressivos mas também encontrados em outros produtos processados, como conservas e alimentos prontos para consumo (Tabelas 1 e 2). Estatísticas provenientes da Irlanda e da Inglaterra indicam que as carnes processadas ou curadas respondem por aproximadamente $20 \%$ da oferta de sódio da dieta (DESMOND, 2006).

O NaCl constitui a principal fonte de sódio nos alimentos, embora outros aditivos (em menor quantidade) também contribuam para aportá-lo nos produtos cárneos, como os sais sódicos: ascorbato, lactato, acetato, citrato, fosfato e glutamato (VANDEDRIESSCHE, 2008). Esse último apresenta crescente uso na indústria alimentícia como realçador de sabor, objetivando melhorar os aspectos sensoriais de produtos à base de carnes, peixes, frutos do mar, vegetais, molhos, temperos e sopas (SHIBATA et al., 1995). Outro sal acrescentando em diminutas concentrações, mas de forma igualmente frequente, é o eritorbato sódico utilizado como antioxidante na maioria dos produtos cárneos industrializados (BRASIL, 1998; BRANEN et al., 2002). 
TABELA 1 - CONCENTRAÇÃO DE SÓDIO EM PRODUTOS NÃO CÁRNEOS

\begin{tabular}{l|c}
\hline Alimento & g sódio $/ 100$ g de alimento \\
\hline Macarrão instantâneo & $1,516 \mathrm{~g}$ \\
\hline Pão francês & $0,648 \mathrm{~g}$ \\
\hline Batata chips & $0,607 \mathrm{~g}$ \\
\hline Pão de queijo assado & $0,773 \mathrm{~g}$ \\
\hline Extrato de tomate & $0,498 \mathrm{~g}$ \\
\hline Queijo parmesão & $1,844 \mathrm{~g}$ \\
\hline Azeitona verde & $1,347 \mathrm{~g}$ \\
\hline Paçoca & $0,167 \mathrm{~g}$ \\
\hline Biscoito maisena & $0,352 \mathrm{~g}$ \\
\hline Biscoito recheado & $0,235 \mathrm{~g}$ \\
\hline Cereal matinal de milho & $0,655 \mathrm{~g}$ \\
\hline
\end{tabular}

Adaptado de TACO (2006).

TABELA 2 - CONCENTRAÇÃO DE SÓDIO EM PRODUTO CÁRNEOS

\begin{tabular}{l|c}
\hline \multicolumn{1}{c|}{ Produto } & g sódio/100 g de produto \\
\hline Almôndega $^{1}$ & $0,62 \mathrm{~g}$ \\
\hline Charque $^{2}$ & $5,85 \mathrm{~g}$ \\
\hline Embutidos fermentados $^{3}$ & $1,5 \mathrm{~g}$ \\
\hline Hambúrguer bovino $^{1}$ & $0,87 \mathrm{~g}$ \\
\hline Linguiça de frango $^{1}$ & $1,13 \mathrm{~g}$ \\
\hline Linguiça suína $^{1}$ & $1,18 \mathrm{~g}$ \\
\hline Presunto cozido $^{3}$ & $0,9 \mathrm{~g}$ \\
\hline Presunto maturado $^{3}$ & $2,2 \mathrm{~g}$ \\
\hline Salsicha $^{2}$ & $0,9 \mathrm{~g}$ \\
\hline
\end{tabular}

${ }^{1}$ Adaptado de TACO (2006); ${ }^{2}$ Valores estimados em charque não dessalgado (SHIMOKOMAKI et al., 1998); ${ }^{3}$ VANDENDRIESSCHE (2007).

A Agência Nacional de Vigilância Sanitária (ANVISA), por meio da RDC 24/2010, regulamentou a promoção de informações à população sobre alimentos que contém proporções elevadas de açúcar, gordura trans, sódio ou bebidas com baixo teor nutricional. Essa medida tem por objetivo conscientizar a população sobre os prejuízos à saúde que acompanham o consumo 
excessivo desses nutrientes. A referida Resolução define alimento com elevada concentração de sódio os produtos com quantidade igual ou superior a $400 \mathrm{mg}$ de sódio por $100 \mathrm{~g}$ ou $100 \mathrm{~mL}$ (BRASIL, 2010).

\subsection{AÇÃO HIPERTENSIVA DO SÓDIO}

Hipertenso é o individuo que apresenta níveis elevados de pressão arterial sustentados acima dos $140 \mathrm{mmHg}$ para pressão sistólica e $90 \mathrm{mmHg}$ para diastólica. A hipertensão arterial sistêmica representa a mais prevalente doença arterial em nível mundial e principal causa de morte no Brasil (LESSA, 2010). O número de hipertensos corresponde à aproximadamente $30 \%$ da população brasileira com mais de vinte anos (SBC, 2010).

A mortalidade resultante de doenças cardiovasculares aumenta progressivamente com a elevação da pressão arterial e está intimamente relacionada às alterações metabólicas e modificações funcionais e estruturais dos órgãos. A elevação da pressão arterial não tratada pode evoluir para complicações vasculares como insuficiência renal ou cardíaca, acidente vascular hemorrágico e complicações de natureza aterosclerótica, como acidente vascular cerebral áterotrombótico, doença cardíaca coronariana e doença arterial periférica (SIMÕES e SCHMIDT, 1996).

Diversos fatores estão relacionados à elevação da pressão arterial, dentre os quais se destaca o consumo elevado de sal. O sódio influencia o sistema vascular promovendo vasoconstrição e maior retenção de líquidos pelos rins, resultando no desenvolvimento da hipertensão arterial (BARROSO, ABREU e FRANCISCHETTI, 2002; RIGATTO, BÖHLKE e IRIGONYEN, 2004; ANTONELLO, ANTONELLO e LOS SANTOS, 2007). Entretanto, além do consumo de sódio, a sensibilidade do indivíduo ao desenvolvimento da hipertensão está associada a outros fatores, dentre os quais se encontram a predisposição genética, sendo mais evidente em negros e indivíduos com histórico familiar positivo, sedentários, obesos e idosos (SBC, 2010)

As evidências relacionando o consumo excessivo de sal ao desenvolvimento de doenças crônicas, como a hipertensão, norteou instituições como a Sociedade Brasileira de Hipertensão, Food Standarts Agency (Reino Unido), Food Safety Authority of Ireland (Irlanda), o Institute of Medicine e a National Academy of Science (Estados Unidos da América) a recomendarem a redução global da ingestão salina para valores de 6 gramas de sal, o equivalente ao limite de $2,4 \mathrm{~g}$ diários de sódio (SBC, 2010). Em 2005, a Food Standarts Agency do Reino Unido estabeleceu plano gestor orientando indústrias alimentícias a reduzirem a concentração salina nos alimentos até o ano de 2010. Tais medidas na indústria cárnea, por exemplo, limitam a concentração de sal em 3,0\% para bacon, 2,5\% em presuntos e 1\% em hambúrgueres (DESMOND, 2006).

\subsection{APLICAÇÕES TECNOLÓGICAS DO NaCL EM PRODUTOS CÁRNEOS NA PROMOÇÃO DO GOSTO SALGADO}

A capacidade de ofertar gosto salgado aos alimentos tornou-se a aplicação tecnológica mais conhecida do cloreto de sódio. A percepção do salgado é possível graças à presença de canais iônicos específicos para os íons $\mathrm{Na}^{+}$e $\mathrm{Li}^{2+}$, presentes na membrana apical de células receptoras situadas na língua. Quando permeados por esses cátions, tais receptores transmitem sinais para o cérebro promovendo a percepção do salgado. Outros íons, como o potássio, por não se adequarem a esses canais não propiciam pelo mesmo mecanismo a percepção do gosto salgado pelos indivíduos (RUUSUNEN, TIRKKONEN e PUOLANNE, 2001).

Estudos demonstraram que a concentração lipídica influencia a percepção do gosto salgado em produtos cárneos. Ruusunen, Tirkkonen e Puolanne (2001) por meio de avaliação sensorial, envolvendo 14 julgadores treinados, observaram aumento na percepção do salgado em salsichas tipo Bologna de acordo com a elevação da concentração lipídica de 8 a $28 \%$, independentemente da 
formulação apresentar 1,2 ou $2 \%$ de $\mathrm{NaCl}$. Concentrações superiores de gordura em preparações de carne moída também proporcionaram maior percepção do gosto salgado. Experimentos para avaliar o efeito do aumento da proporção de carne magra de 50 para $60 \%$ em almôndegas, quando mantidos os níveis de $\mathrm{NaCl}$, resultaram em menor percepção do salgado. Por outro lado, nesse mesmo estudo, a elevação do teor de gordura de $10 \%$ para $20 \%$, utilizando-se igualmente $400 \mathrm{mg}$ $\mathrm{NaCl} / 100 \mathrm{~g}$, promoveu maior percepção do salgado medida em escala de 0 a 10, com evolução média de 4,2 para 4,8 (RUUSUNEN e PUOLANNE, 2005).

A identificação do gosto salgado também sofre influência da rotina dos níveis de $\mathrm{NaCl}$ comumente presentes na dieta. A redução de sua concentração durante algum tempo é acompanhada de novo patamar de percepção pelos consumidores sem que ocorram prejuízos significativos à aceitação do produto (VANDENDRIESSCHE, 2008). No entanto, a redução do sal na dieta deve ser gradativa (ao longo de meses) para que a aceitabilidade dos alimentos não seja comprometida (BERTINO, BEAUCHAMP e ENGELMAN, 1982; DESMOND, 2006).

\subsection{EFEITO DO SAL SOBRE A ATIVIDADE DE ÁGUA E ESTABILIDADE MICROBIOLÓGICA}

Charque, jerked beef, presuntos maturados, miúdos e retalhos salgados de suínos são exemplos de produtos cárneos cuja conservação resulta principalmente da salga e independem de refrigeração (SHIMOKOMAKI et al., 1998; SHIMOKOMAKI, YOUSSEF e TERRA, 2003; ROCHA GARCIA et al., 2010). A função conservante nos produtos cárneos pode ser justificada pelo efeito do sal sobre a redução da atividade de água ( $\mathrm{Aa}$ ), além da ação antimicrobiana proporcionada diretamente pelos íons $\mathrm{Na}^{+}$(VARNAM e SUTHERLAND, 1998; VAZ, 2005).

Estudo avaliando a capacidade de desenvolvimento de Staphylococcus aureus e Clostridium botulinum durante o processamento do charque ilustra a ação conservante do sal em produtos cárneos (LARA et al., 2003). Ao término do processo, as amostras apresentaram Aa entre 0,70 e 0,75 e pH 5,30 a 5,60, condições que impedem a proliferação do Clostridium botulinum (HAUSCHILD e DOODS, 1993). A contagem de Clostridium botulinum após 60 dias de armazenamento mantevese em 100 células viáveis/g, número semelhante ao observado no início do processo, indicando que as drásticas condições para obtenção do produto inibem a germinação de esporos e, por consequência, a produção de toxinas. A avaliação de Staphylococcus aureus demonstrou que as etapas de obtenção do charque proporcionam ambiente igualmente desfavorável à proliferação desse micro-organismo. Encerrada a etapa de secagem ao sol, a contagem microbiana foi reduzida de 5,4 $\times 10^{5}$ UFC para 10,0 $\times 10^{2}$ UFC demonstrando que a salga e a desidratação resultam em obstáculos significativos para a proliferação desse micro-organismo.

\subsection{EFEITO DO NaCL SOBRE AS PROPRIEDADES FUNCIONAIS DAS PROTEÍNAS CÁRNEAS}

Soluções salinas apresentam a capacidade de promover a solubilização de proteínas miofibrilares, como actina e miosina (SHIMOKOMAKI et al., 2006). Essa ação assume singular importância tecnológica para que essas macromoléculas possam formar géis, atuar como emulsificantes, melhorar a capacidade de retenção de água e gordura, além de prover maciez aos produtos cárneos (SHIMOKOMAKI et al., 2006; ROCHA GARCIA et al., 2007; VANDENDRIESSCHE, 2008).

Os primeiros estudos avaliando o efeito do sal sobre proteínas cárneas apontavam a capacidade do cloreto de sódio (em pequenas concentrações) estabilizar proteínas por meio de interações eletrostáticas, mediadas pela força iônica do meio (TANFORD, 1961). Arakawa e Timasheff (1984) demonstraram que soluções salinas exercem efeitos específicos sobre as proteínas conforme a concentração e natureza do sal, podendo estabilizar ou promover a desnaturação, causando os efeitos descritos, respectivamente, como salting-in ou salting-out. Cheftel, Cuq e Lorient (1989), relataram que a concentração iônica influencia a capacidade de absorção de água e a solubilidade de proteínas 
cárneas, proporcionando maior solvatação e assim caracterizando o efeito salting-in. Por outro lado, a crescente concentração salina do meio faz com que interações água-sal superem a afinidade entre a água e a proteína, reduzindo a solubilidade e resultando na ocorrência do efeito salting-out.

Segundo Offer e Knight (1988) e Offer e Trinick (1983), o íon sódio ( $\left.\mathrm{Na}^{+}\right)$interage com as proteínas formando uma nuvem eletrônica ao redor dos filamentos de actina e miosina. Por outro lado, Hamm (1986) propôs que o cloro é o principal responsável pela mediação das interações entre a água e as proteínas cárneas. Por se tratar de ação iônica de natureza eletrostática, os íons cloro que apresentam maior afinidade pelas proteínas miofibrilares (quando comparados ao sódio) ligam-se às regiões de carga positiva das moléculas de miosina, aumentando as cargas negativas das proteínas e causando repulsão entre os pólos com semelhança eletrostática. Essa ação, acompanhada pela redução do ponto isoelétrico para pH inferior, minimiza a interação entre os grupos de cargas opostas presentes nas proteínas. Embora restrita, sobretudo às proteínas do filamento de miosina, essa ação faz com que o eixo da estrutura das moléculas miofribilares seja comprometido e ocorra intumescimento resultante da interação entre as proteínas miofibrilares e a água presente no meio (HAMM, 1986).

Diversos trabalhos identificaram diferenças entre os efeitos promovidos por sais mono e divalentes sobre as proteínas miofibrilares (LUVIELMO e ANTUNES, 2006). Compostos iônicos divalentes, como cloreto de cálcio $\left(\mathrm{CaCl}_{2}\right)$ ou magnésio $\left(\mathrm{MgCl}_{2}\right)$, formam ligações iônicas entre os grupos carboxílicos adjacentes presentes nas proteínas (SMITH e ROSE, 1994). A maior carga eletrostática nos sais divalentes amplia as interações com a matriz cárnea e limita a difusão dos sais propiciando a redução da atividade de água do produto (ALIÑO et al., 2010a).

Os sais influenciam a hidratação de proteínas aumentando a capacidade de retenção de água e, por consequência, a textura e rendimento dos processos (SHIMOKOMAKI et al., 2006; ROCHA GARCIA et al., 2010). Amostras de patinho traseiro apresentaram aumento na capacidade de retenção de água e ganho de massa quando tratadas com soluções contendo de $3 \%$ a $15 \%$ de $\mathrm{NaCl}$, combinadas ao uso individual dos hidrocoloides $(0,5 \%)$ carragena, pectina ou proteína isolada de soja (ROCHA GARCIA et al., 2010). Nos produtos cárneos emulsionados, como mortadelas e salsichas, as proteínas miofibrilares solúveis em soluções salinas, bem como as moléculas de colágeno, formam filme ao redor das moléculas de gordura estabilizando-as durante os processos que envolvem tratamentos térmicos (MONAHAN e TROY, 1997; OLIVO e SHIMOKOMAKI, 2001; DESMOND, 2006).

\subsection{ALTERNATIVAS PARA REDUÇÃO DO NaCL EM PRODUTOS CÁRNEOS}

Motivada pela crescente preocupação dos consumidores em relação ao consumo excessivo de sódio, a indústria busca alternativas para redução dos níveis de $\mathrm{NaCl}$ nos alimentos. Porém, minimizar as concentrações de sódio nos produtos cárneos excede as alternativas para contornar prejuízos ao gosto salgado e exige o desenvolvimento de inovações tecnológicas capazes de substituir as propriedades funcionais promovidas pelo sal (RUUSUNEN et al., 2005; SHIMOKOMAKI et al., 2006).

As alternativas para a diminuição do sódio nas carnes processadas envolvem a redução direta do teor de sal adicionado, a substituição total ou parcial do $\mathrm{NaCl}$ por sais não sódicos, modificações no processo ou a combinação desses mecanismos (TERREL, 1983; SOFOS, 1984; SOFOS, 1986; RUUSUNEN et al., 2005). O uso de aditivos ou modificação de ingredientes também são métodos utilizados para contornar as propriedades funcionais proporcionadas pelo sal, como a promoção da coesão de fragmentos cárneos e textura (RUUSUNEN et al., 2005; LÓPEZ-LÓPEZ et al., 2010).

\subsection{SUBSTITUIÇÃO DO NACL POR SAIS NÃO SÓDICOS}

A redução do teor de sódio nos produtos cárneos pode ser alcançada pela substituição do 
$\mathrm{NaCl}$ por outros sais não sódicos. A Organização das Nações Unidas para Alimentos e Agricultura (FAO), por meio do Comitê de Especialistas em Aditivos Alimentares, classificou um grupo de substâncias como substituintes do sal. Estão incluídos nessa classe o sulfato de potássio, o ácido glutâmico e seus sais, como o glutamato de amônio, magnésio, potássio e cálcio, sendo excluído desse grupo seu respectivo sal-sódico que (embora apresente menor proporção de sódio quando comparado ao $\mathrm{NaCl}$ ) pode ser considerado como significativa fonte de sódio, principalmente em razão do seu uso como realçador de sabor (FAO, 1981; BRANEN et al., 2002).

A substituição do íon sódio por magnésio ou potássio é limitada em virtude desses íons ofertarem gosto amargo aos alimentos. Pode-se exemplificar tal dificuldade na substituição do cloreto de sódio pelo similar contendo potássio, alternativa acompanhada de maior custo e comprometimento da palatabilidade, limitando o uso do chamado "sal-light" por hipertensos (LOTAIF et al., 1995). O "sal-light", produto composto por $50 \%$ de cloreto de sódio e $50 \%$ de cloreto de potássio, é indicado para indivíduos que seguem dieta com redução de teores de sódio podendo ser utilizado na dietoterapia da hipertensão arterial sistêmica (PAULINO et al., 2006; ASSUNÇÃO, 2006). As deficiências de gosto podem ser minimizadas quando se efetua a substituição utilizando combinações de diferentes sais (VANDENDRIESSCHE, 2008).

Experimentos investigaram o efeito da substituição do $\mathrm{NaCl}$ por outros sais sobre as características de presuntos maturados. Foram avaliadas duas combinações, uma formada por compostos monovalentes $(50 \% \mathrm{KCl}$ e $50 \% \mathrm{NaCl})$ e outra formada por $\mathrm{KCl}(25 \%), \mathrm{CaCl}_{2}(15 \%)$, $\mathrm{MgCl}_{2}(5 \%)$ e $\mathrm{NaCl}(55 \%)$, sendo o controle elaborado com $100 \%$ de $\mathrm{NaCl}$. A adição de $\mathrm{CaCl}_{2} \mathrm{e}$ $\mathrm{MgCl}_{2}$ resultou em menor concentração salina no produto final e exigiu maior tempo para redução da atividade de água. Embora não tenham sido evidenciadas diferenças sensoriais, os resultados demonstraram que a substituição do $\mathrm{NaCl}$ por outros sais deve ser acompanhada de rígido controle. Substitutos divalentes, como $\mathrm{CaCl}_{2}$ e $\mathrm{MgCl}_{2}$, em razão do maior número de cargas positivas interagem de forma mais acentuada com as proteínas cárneas, encontrando maior dificuldade para penetrar na estrutura cárnea e exigem maior tempo de processo para que seja alcançada a redução da atividade de água e umidade, quando comparada a sais monovalentes como o $\mathrm{NaCl}$ (ALIÑO et al., 2010b).

Zanardi et al. (2010) avaliaram a substituição parcial do $\mathrm{NaCl}$ pela mistura de $\mathrm{KCl}, \mathrm{CaCl}_{2} \mathrm{e}$ $\mathrm{MgCl}_{2}$ em salames italianos e observaram que a redução de $50 \%$ de $\mathrm{NaCl}$ não ocasionou diferenças no pH, atividade de água e teor de ácidos graxos livres do produto em relação ao salame tradicional, mas induziu aumento significativo da sua oxidação lipídica.

O efeito da redução do sal sobre os parâmetros físico-químicos de lombos curados foi avaliado por Aliño et al. (2010a). Nesse experimento, o teor de $\mathrm{NaCl}$ foi parcialmente substituído por combinações de $\mathrm{KCl}, \mathrm{CaCl}_{2}$ e $\mathrm{MgCl}_{2}$ em quatro diferentes proporções. A substituição de $70 \%$ do $\mathrm{NaCl}$ pelas combinações aumentou a firmeza e mastigabilidade do produto. O conteúdo de cloro, umidade e cor não foram influenciados pelo uso das diferentes combinações dos outros sais, porém houve maior dispersão de potássio nas amostras em comparação ao cálcio, magnésio e sódio. Costa-Corredor et al. (2009) verificaram que presuntos curados com redução do teor de sal de $3 \%$ para 1,5\% apresentaram diminuição do gosto salgado, aumento da atividade de água, maior proteólise e maciez, porém esses efeitos foram minimizados pela adição de $2 \%$ de lactato. Os sais substitutos do cloreto de sódio podem promover efeito semelhante sobre a atividade de água, porém acarretam prejuízo sensorial aos produtos cárneos (VANDENDRIESSCHE, 2008).

\subsection{MODIFICAÇÃO DE INGREDIENTES E PROCESSOS NA FORMULAÇÃO DE PRODUTOS CÁRNEOS COM REDUZIDA CONCENTRAÇÃO SALINA}

Nos países do sul do mediterrâneo são produzidos presuntos com elevado teor de sal (11\%), cujo período de salga varia de 3 a 4 dias $/ \mathrm{kg}$ de presunto e o período de maturação requer aproximadamente 18 meses. Estudo avaliando o efeito do tempo de salga sobre as características desses presuntos demonstram que produtos obtidos por meio de salga rápida (1-1,5 dia/kg de 
músculo), ao final de 18 meses, apresentaram menor concentração de cloretos, maior umidade e superior concentração de ácidos graxos poliinsaturados quando comparados aos produtos obtidos por meio de salga lenta (4-5 dias/kg de carne). Esses resultados evidenciam que a redução de sal nos presuntos curados pode ser obtida pela diminuição do tempo de salga, com a vantagem de preservar a concentração de ácidos graxos poliinsaturados com maior valor nutricional (COUTRONGAMBOTTI, 1999).

Ruusunen e Puolanne et al. (2005) afirmaram que o uso de fosfatos permite reduzir a concentração de sódio de valores entre 1,5 e 1,7\% para 1,4\% em salsichas, sem que ocorra prejuízo sensorial ao produto. A adição de polifosfatos reduz as interações actina-miosina e fragiliza a estrutura miofibrilar facilitando as interações com a água e minimizando a necessidade de $\mathrm{NaCl}$ para a solubilização proteica. O uso de amido de mandioca modificado, combinado a citrato de sódio, também se mostrou efetivo para minimizar as perdas de água e gordura durante o tratamento térmico de salsichas formuladas com reduzido teor de sódio (RUUSUNEN et al., 2003).

A redução do teor de sal pode ser acompanhada de prejuízo à estrutura dos produtos cárneos. Nos embutidos, as concentrações de $\mathrm{NaCl}$ devem respeitar o mínimo de 2,25\% para evitar prejuízos ao seu sabor e firmeza (RUUSUNEN et al., 2005). Presuntos cozidos também exemplificam a importância do $\mathrm{NaCl}$ na atribuição de propriedades funcionais. As concentrações de $\mathrm{NaCl}$ desses produtos devem ser mantidas entre 1,7 e 2\%, menores proporções são acompanhadas de prejuízo à adesão dos fragmentos cárneos e maiores perdas durante o cozimento (RUUSUNEN, SIMOLM e PUOLANE, 2001; DESMOND, 2006).

A associação de algas comestíveis do tipo wakame com emulsão de óleo de oliva foi avaliada como substituinte de toucinho por López-López et al. (2010) em hambúrgueres bovinos formulados com baixo teor de sal $(0,5 \%)$ e de gordura $(<10 \%)$. Nos hambúrgueres em que foi utilizada a combinação ocorreram menores perdas durante o cozimento e maior maciez em relação ao controle. Os autores sugeriram que os resultados positivos associados ao uso das algas se devem ao elevado conteúdo de fibras presente e à capacidade de retenção de água desses ingredientes. O uso de algas para contornar efeitos da redução do teor de sal também foi estudado por Cofrades et al. (2008) que avaliaram individualmente a ação de algas comestíveis Sea Spaghetti (Himanthalia elongata), Wakame (Undaria pinnatifida) e Nori (Porphyra umbilicalis) sobre as características físico-químicas e estruturais de emulsões cárneas formuladas com teor de $\mathrm{NaCl}$ reduzido a 0,5\%. Novamente, a adição das algas proporcionou maior retenção de água e gordura, além de promover maior firmeza aos produtos.

\subsection{CONSERVAÇÃO DE PRODUTOS CÁRNEOS FORMULADOS COM REDUÇÃO DE SAL}

O uso de óxido nítrico combinado à salga mostrou-se eficiente para redução da contagem microbiana em presuntos curados. Após 180 dias de maturação foi verificada redução da Aa para 0,94 em amostras tratadas apenas com sal e 0,91 em amostras em que houve o uso de óxido nítrico. Também foi observada redução das unidades formadoras de colônia de Staphylococcus aureus de 8,12 log UFC/ $\mathrm{cm}^{2}$ para aproximadamente $5 \mathrm{log} \mathrm{UFC} / \mathrm{cm}^{2}$, demonstrando a efetividade do óxido nítrico em propiciar ambiente adverso para o metabolismo e produção de enterotoxinas por parte desse micro-organismo (PORTOCARRERO, NEWMAN e MIKEL, 2002).

Estudo avaliando o efeito do cloreto de potássio sobre bactérias patogênicas (Aeromonas hydrophila, Enterobacter sakazakii, Shigella flexneri, Yersinia enterocolitica e Staphylococcus aureus) demonstrou que esse sal apresenta efeito antimicrobiano equivalente ao $\mathrm{NaCl}$ (BIDLAS e LAMBERT, 2008). O uso de nisina combinada a $\mathrm{NaCl}$ mostrou-se eficiente na redução da contagem microbiana, demonstrando que essa associação pode ser utilizada quando se deseja conservar produtos formulados com redução de sódio (PAWAR et al., 2000). 


\section{CONCLUSÃO}

A preocupação dos consumidores relacionada aos efeitos prejudiciais à saúde, associada ao consumo excessivo de sódio, exigirá das indústrias alimentícias continua redução do uso de sal nos alimentos. Essa limitação enfrenta maior complexidade no segmento cárneo, pois além de prover palatabilidade, o $\mathrm{NaCl}$ é responsável por diversas propriedades funcionais. A busca de alternativas para redução do uso de $\mathrm{NaCl}$, sem prejuízo para a qualidade sensorial dos produtos, constitui desafio a ser enfrentado pela indústria, visando manter a aceitação e consumo dos derivados cárneos.

\section{ABSTRACT \\ TECHNOLOGY APPLICATIONS AND ALTERNATIVES TO REDUCE SODIUM CHLORIDE IN MEAT PRODUCTS}

The aim of this study was to review the applications and technological alternatives for reducing the use of sodium chloride in meat products. This review have approached the characteristics of consumption of sodium chloride, highlighting its hypertensive action, the addition of sodium chloride in meat products, addressing the promotion of salty taste, effect on water activity and microbiological stability, as well as its influence on the functional properties of meat proteins. Some alternatives methods to reduce the levels of sodium chloride in meat products, such as use of non-sodium salts, new ingredients and alternative methods of preservation were also pointed out. The consumers' concern related to adverse health effects associated with excessive sodium consumption will require that the food industries keep reducing the use of salt in food. This restriction affects meat products in a very complex way because, in addition to promote palability, the sodium chloride is responsible for many functional properties of these products. The search for alternatives to reduce de utilization of sodium chloride without compromising the sensory quality of meat products is a challenge to be faced by the industries in order to maintain the acceptance and consumption of these products.

KEY-WORDS: MEAT; FUNCTIONAL PROPERTIES; HYPERTENSION; NaCI; SALT SUBSTITUTE.

\section{REFERÊNCIAS}

1 ALIÑO, M.; GRAU, R.; TOLDRÁ, F.; BLESA, E.; PAGÁN, J.M.; BARAT, J. M. Physicochemical properties and microbiology of dry-cured loins obtained by partial sodium replacement with potassium, calcium and magnesium. Meat Science, v.85, p.580-588, 2010a.

2 ALIÑO, M.; GRAU, R.; TOLDRÁ, F.; BARAT, J.M. Physicochemical changes in dry-cured hams salted with potassium, calcium and magnesium chloride as a partial replacement for sodium chloride. Meat Science, v.86, p.331-336, 2010b

3 ANTONELLO, V.S.; ANTONELLO, I.C.F.; LOS SANTOS, C.A. Sensibilidade gustativa ao sal, natriúria e pressão arterial em indivíduos normotensos. Revista da Associação Médica Brasileira, v. 53, n. 2, p. 142-146, 2007.

4 ARAKAWA, T.; TIMASHEFF, S.N. Mechanism of protein salting in and salting out by divalent cation salts: balance between hydration and salt binding. Biochemistry, v. 23, p. 5912-5923, 1984.

5 ASSUNÇÃO, A.M.P. Hipocalemia em pacientes com a síndrome da resposta de fase aguda. 2006. 58 f. Dissertação (Mestrado em Patologia Clínica), Universidade Federal do Triângulo Mineiro, Uberaba, 2006.

6 BARROSO, S.G.; ABREU, V.G.; FRANCISCHETTI, E.A. A participação do tecido adiposo visceral na gênese da hipertensão e doença cardiovascular aterogênica. Um conceito emergente. Arquivo Brasileiro de Cardiologia, v. 78, n. 6, p. 618-630, 2002.

7 BERTINO, M.; BEAUCHAMP, G.K.; ENGELMAN, K. Long-term reduction in dietary sodium alters the taste of salt. The American Journal of Clinical Nutrition, v. 36, p. 1134-1144, 1982.

8 BIDLAS, E.; LAMBERT, R.J.W. Comparing the antimicrobial effectiveness of $\mathrm{NaCl}$ and $\mathrm{KCl}$ with a view to salt/sodium replacement. International Journal of Food Microbiology, v. 124, p. 98-102, 2008.

9 BRANEN, A.L; DAVIDSON, P.M.; SALMINEN S.; THOMGATE, J.H. Food additives. $2^{\text {nd }}$ ed. New York: Marcel Dekker, 2002.

10 BRASIL. Secretaria de Vigilância Sanitária. Portaria n. 1004 de 14 de dezembro de 1998. Regulamento técnico para atribuição de função de aditivos e seus limites máximos para a categoria 8 - carne e produtos cárneos. Diário Oficial [da] República Federativa do Brasil, Brasília, 11 de dezembro de 1998, Seção 1, p. 28. 
11 BRASIL. Agência Nacional de Vigilância Sanitária. Resolução n. 24 de 29 de junho de 2010. Dispõe sobre a oferta, propaganda, publicidade, informação e outras práticas correlatas cujo objetivo seja a divulgação e a promoção comercial de alimentos considerados com quantidades elevadas de açúcar, de gordura saturada, de gordura trans, de sódio, e de bebidas com baixo teor nutricional. Diário Oficial [da] República Federativa do Brasil, Brasília, 15 de junho de 2010 , Seção 1, p. 46

12 CHEFTEL, J.C.; CUQ, J.L.; LORIENT, D. Proteínas alimentarias. Zaragoza: Acribia, 1989. p. 179-220; $291-335$.

13 COFRADES, S.; LÓPEZ-LÓPEZ, I.; SOLAS, M.T.; BRAVO, L.; JIMÉNEZ-COLMENERO, F. Influence of different types and proportions of added edible seaweeds on characteristics of low-salt gel/emulsion meat systems. Meat Science, v. 79, p. 767-776, 2008.

14 COSTA-CORREDOR, A.; SERRA, X.; ARNAU, J.; GOU, P. Reduction of NaCl content in restructured dry-cured hams: post-resting temperature and drying level effects on physicochemical and sensory parameters. Meat Science, v. 83, p. 390-397, 2009

15 COUTRON-GAMBOTTI, C.; GANDEMER, G.; ROUSSET, S.; MAESTRINI, O.; CASABIANCA, F. Reducing salt content of dry-cured ham: effect on lipid composition and sensory attributes. Food Chemistry, v. 64, p. 13-19, 1999.

16 DESMOND, E. Reducing salt: a challenge for the meat industry. Meat Science, v. 74, p. 188-196, 2006.

17 Food and Drug Admnistration (FAO). Codex standard for special dietary foods with low-sodium content (including salt substitutes). Roma 1981. 3 p.

18 Food Safety Authority of Ireland (FSAI). Salt and health: review of the scientific evidence and recommendations for public policy in Ireland. Dublin, 2005. 28 p.

19 HAMM, R. Functional properties of the myofibrillar system. In: BECHTEL, P.J. (Ed.). Muscle as food. New York: Academic Press, 1986. p. 135-200.

20 HAUSCHILD, A. W.; DODDS, K. Clostridium botulinum ecology and control in foods. New York: Marcel Dekker, 1993.

21 Instituto Brasileiro de Geografia e Estatística (IBGE). Pesquisa de orçamentos familiares 2008-2009: avaliação nutricional da disponibilidade domiciliar de alimentos no Brasil. Rio de Janeiro, 2010.

22 LARA, J.A.F.; SENIGALIA, S.W.B.; OLIVEIRA, T.C.R.M.; DUTRA, I.S.; PINTO, M.F.; SHIMOKOMAKI, M. Evaluation of survival of Staphylococcus aureus and Clostridium botulinum in charqui meats. Meat Science, v. 65, p. 609-613, 2003.

23 LESSA, I. Hipertensão arterial sistêmica no Brasil: tendência temporal. Cadernos de Saúde Pública, v. 26, n. 8, p. 14701471,2010

24 LÓPEZ-LÓPEZ, I.; COFRADES, S.; RUIZ-CAPILLAS, C.; JIMÉNEZ-COLMENERO, F. Design and nutritional properties of potential functional frankfurters based on lipid formulation, added seaweed and low salt content. Meat Science, v.83, p. 255-262, 2010.

25 LOTAIF, L.A.D; KOHLMANN JR, O.; ZANTELLA, M.T; KOHLMANN, B.; RIBEIRO, A.B. Efeito da suplementação de potássio através do sal de cozinha na hipertensão arterial primária leve e moderada. Jornal Brasileiro de Nefrologia, v. 17, n. 4, p. 214-218, 1995.

26 LUVIELMO, M.M.; ANTUNES, A.J. Substituição de proteínas da carne por proteínas do concentrado protéico de soro e adição de $\mathrm{CaCl} 2$ em sistema cárneo. Boletim do CEPPA, v 24, n. 1, p. 25-46, 2006.

27 MATTES, R.D. The taste for salt in humans. The American Journal of Clinical Nutrition, v. 65, p. 692-697, 1997.

28 MONAHAN, F.J.; TROY, D.J. Overcoming sensory problems in low fat and low salt products. In: PEARSON, A. M.; DUTSON, T. R. (Eds.). Advances in meat research. Production and processing of healthy meat, poultry and fish products. London: Blackie Academic \& Professional, 1997. v. 11, p. 257-281.

29 OLIVO, R.; SHIMOKOMAKI, M. Carnes: no caminho da pesquisa. Cocal do Sul: Imprint, 2001.155 p.

30 OFFER, G.; KNIGHT, P. The structural basis of water-holding in meat. In: LAWRIE, R. A. (Ed.). Developments in meat science. London: Elsevier Applied Science, 1988. v. 4. p. 63-243.

31 OFFER, G.; TRINICK, J. On the mechanism of water-holding in meat: the swelling and shrinking of myofibrils. Meat Science, v. 8, p. 245-281, 1983.

32 PAULINO, F.O.; SILVA, T.J.P.; FRANCO, R.M.; FREITAS, M.Q.; FERNANDES, M.L. Redução parcial dos teores de gordura e sal em embutido cárneo suíno com utilização de goma carragena e cloreto de potássio. Revista Brasileira de Ciências Veterinárias, v. 13, n. 2, p. 121-124, 2006. 
33 PAWAR, D.D.; MALIK, S.V.S.; BHILEGAONKAR, K.N.; BARDUDDHE, S.B. Effect of nisin and its combination with sodium chloride on the survival of Listeria monocytogenes added to raw buffalo meat mince. Meat Science, v. 56, p. 215-219, 2000 .

34 PORTOCARRERO, S.M.; NEWMAN, M.; MIKEL, B. Staphylococcus aureus survival, Staphylococcal enterotoxin production and shelf stability of country-cured hams manufactured under different processing procedures. Meat Science, v. 62, p. $267-273,2002$.

35 RIGATTO, K.V.; BÖHLKE, M.; IRIGOYEN, M.C. Sistema renina angiotensina: da fisiologia ao tratamento. Revista da Sociedade de Cardiologia do Rio Grande do Sul, n. 3, p.1-5, 2004.

36 ROCHA GARCIA, C.E.; YAMASHITA, F; YOUSSEF, E. Y.; SHIMOKOMAKI, M. Role of carrageenan in intermediate moisture meat products processed under high salt concentration. In: INTERNATIONAL CONGRESS OF MEAT SCIENCE AND TECHNOLOGY, 53 ${ }^{\text {th }}$, Beijing, 2007. Proceedings... Beijing: Elsevier, 2007. v. 53.

37 ROCHA GARCIA, C.E.; BOLOGNESI, V.J.; CASTILHO, M.P.; NASCIMENTO, A.J.; MASSON, M.L. Rendimento e parâmetros físico-químicos de charque elaborado com soluções salinas de hidrocolóides. In: SIMPÓSIO DE CIÊNCIA E TECNOLOGIA DE ALIMENTOS, 2., Aracaju. 2010. Anais ... Aracaju: SBCTA-SERGIPE, 2010. p. 1628-1631.

38 RUUSUNEN, M.; SIMOLM, M.; PUOLANNE, E. The effect of fat content and flavor enhancers on the perceived saltiness of cooked 'bologna type' sausages. Journal of Muscles Food, v.12, p.107-120, 2001.

39 RUUSUNEN, M.; TIRKKONEN, M.S.; PUOLANNE, E. Saltiness of coarsely ground cooked ham with reduced salt content. Agricultural and Food Science in Finland, v. 10, p. 27-32, 2001.

40 RUUSUNEN, M.; VAINIONPÄÄ, J.; PUOLANNE, E.; LYLY, M.; LÄHTEENMÄKI, L.; NIEMISTÖ, M.; AHVENAINEN, R. Physical and sensory properties of low-salt phosphate-free frankfurters composed with various ingredients. Meat Science, v. 63, p. 9-16, 2003.

41 RUUSUNEN, M.; PUOLANNE, E. Reducing sodium intake from meat products. Meat Science, v. 70, p. 531-541, 2005.

42 RUUSUNEN, M.; VAINIONPÄÄ, J.; LYLY, M.; LÄHTEENMÄKI, L.; NIEMISTÖ, M.; AHVENAINEN, R.; PUOLANNE, E. Reducing the sodium content in meat products: the effect of the formulation in low-sodium ground meat patties. Meat Science, v. 69, p. 53-60, 2005.

43 Scientific Advisory Committee on Nutrition (SACN). Salt and health. Norwich: The Stationery Office, 2003.134 p.

44 SARNO, F.; CLARO, R.M.; LEVY, R.B.; BANDONI, D.H.; FERREIRA, S.R.G.; MONTEIRO, C.A. Estimativa de consumo de sódio pela população brasileira. Revista de Saúde Pública, v. 43, n. 2, p. 219-225, 2009.

45 SHIBATA, M.A.; TANAKA, H.; KAWABE, M.; SANO, M.; HAGIWARA, A.; SHIRAI, T. Lack of carcinogenicity of monosodium L-glutamate in fischer 344 rats. Food and Chemical Toxicology, v.33, n. 5, p. 383-391, 1995.

46 SHIMOKOMAKI, M.; FRANCO, B.D.M.; CARVALHO, B.C. Charque e produtos afins: tecnologia e conservação - uma revisão. Boletim da SBCTA, v. 21, n. 1, p. 25-35, 1987.

47 SHIMOKOMAKI, M.; YOUSSEF, E.Y.; TERRA, N.N. Curing. In: CABALLERO, B.; TRUGO, L.; FINGLAS, P. M. Encyclopedia of food sciences and nutrition. $2^{\text {nd }}$ ed. London: Elsevier, 2003. p. 1702-1708,

48 SHIMOKOMAKI, M.; OLIVO, R.; TERRA, N.N.; FRANCO, B.D.G.M. Atualidades em ciência e tecnologia de carnes. São Paulo: Livraria Varela, 2006. 230 p.

49 SHIMOKOMAKI, M.; FRANCO, B.D.G.M.; BISCONTINI, T.M.; PINTO, M.F.; TERRA, N.N.; ZORN, T.M.T. Charqui meats are hurdle technology meat products. Food Reviews International, New York, v.14, n.4, p. 339-349, 1998.

50 SIMÕES, M.V.; SCHMIDT, A. Hipertensão arterial como fator de risco para doenças cardiovasculares. Medicina Ribeirão Preto, v. 29, p. 214-219, 1996.

51 SMITH, D.M.; ROSE, A.J. Gel properties of whey protein concentrates as influenced by ionized calcium. Journal of Food Science, v. 59, n. 5, p. 1115-1118, 1994.

52 SOFOS, J.N. Antimicrobial effects of sodium and other ions in foods: a review. Journal of Food Safety, v. 6, n. 1, p. 45$78,1984$.

53 SOFOS, J.N. Use of phosphates in low-sodium meat products. Food Technology, v. 40, n. 9, p. 52-68, 1986.

54 Sociedade Brasileira de Cardiologia (SBC). Sociedade Brasileira de Hipertensão. Sociedade Brasileira de Nefrologia. VI Diretrizes Brasileiras de Hipertensão. Arquivo Brasileiro de Cardiologia, v. 95, n. 1, p. 1-51, 2010.

55 TABELA brasileira de composição de alimentos (TACO). 2. ed. Campinas: NEPA-UNICAMP, 2006.113 p.

56 TANFORD, C. Physical chemistry of macromolecules. New York: Wiley, 1961. 
57 TERRELL, R.N. Reducing the sodium content of processed meats. Food Technology, v.37, n.7, p.66-71, 1983.

58 U.S. Department of Health and Human Services. U.S. Department of Agriculture. Dietary guidelines for americans. $6^{\text {th }}$ ed. Washington, DC, 2005.

59 VANDENDRIESSCHE, F. Meat products in the past, today and in the future. Meat Science, v. 78, p. 104-113, 2008.

60 VARNAM, A.H.; SUTHERLAND, J.P. Carne y productos cárnicos: tecnología química y microbiología. Zaragoza: Acribia, 1998. p. 423.

61 VAZ, S.K. Elaboração e caracterização de linguiça fresca "tipo toscana" de tilápia (Oreochromis niloticus). 2005. 97 f. Dissertação (Mestrado em Tecnologia dos Alimentos), Universidade Federal do Paraná, Curitiba, 2005.

62 World Health Organization (WHO). Report of a Joint WHO/FAO Expert Consultation. Diet, nutrition and the prevention of chronic diseases. Geneva (Suíça), 2003.

63 ZANARDI, E.; GHIDINI, S.; CONTER, M.; LANIERI, A. Mineral composition of Italian salami and effect of partial replacement on compositional, physico-chemical and sensory parameters. Meat Science, v. 86, n. 3, p. 742-747, 2010. 